\title{
A future for PET imaging in Alzheimer's disease
}

\author{
Aurélie Kas $^{1,2}$ (D) $\cdot$ Raffaella Migliaccio ${ }^{3,4}$ (D) $\cdot$ Bertrand Tavitian $^{5,6}$ (D) \\ Published online: 19 December 2019 \\ (C) Springer-Verlag GmbH Germany, part of Springer Nature 2019
}

\section{The complexity of early-onset Alzheimer's disease}

Alzheimer's disease is defined neuropathologically by abnormal extra-cellular $\beta$-amyloid plaques combined with intraneuronal tau aggregation (neurofibrillary tangles). Observations showing that patients with different clinical presentations and evolutions share the same neuropathological features have led to the notion of an Alzheimer's disease spectrum encompassing typical and atypical forms of Alzheimer's disease $[1,2]$.

In more than $80 \%$ of the cases, Alzheimer's disease is a late-onset disease (arbitrarily defined as patients aged 65 years old or more) with deficits of episodic memory. A dementia prodromal stage called mild cognitive impairment (MCI) aggravates progressively over several years while extending to the language, visuospatial, praxis, and executive domains. In contrast, the less frequent earlyonset (<65 years old) Alzheimer's disease patients usually present at diagnosis with a more dramatic multidomain cognitive impairment involving memory, attention, language, visuospatial, and executive deficits. Unlike the typical form in elderly, MCI rarely precedes serious cognitive impairment in early-onset Alzheimer's disease patients, who often also show a faster evolution towards

Aurélie Kas and Raffaella Migliaccio contributed equally to this work.

This article is part of the Topical Collection on Editorial

Bertrand Tavitian

bertrand.tavitian@inserm.fr

Aurélie Kas

aurelie.kas@gmail.com

Raffaella Migliaccio

lara.migliaccio@gmail.com

1 Nuclear Medicine Department Pitié-Salpêtrière Hospital, APHP Sorbonne-Université, Paris, France

2 Laboratoire d'Imagerie Biomédicale, Sorbonne Université, Paris, France severe stages. Moreover, a large proportion of earlyonset Alzheimer's disease patients present "atypical" clinical forms, with predominant and/or isolated deficits of language, visuospatial, motor, or executive/behavioural functions [2].

In the past decade, pioneering anatomopathological studies [1, 3] and the use of cerebrospinal and imaging biomarkers for Alzheimer's disease have increased emphasis on the phenotypic variability of early-onset Alzheimer's disease. Typical Alzheimer's disease patients show medial temporal atrophy and mild-to-moderate posterior brain hypometabolism, while in atypical variants, extensive posterior cortical damage stands in contrast with the relative sparing of the medial temporal lobe. The distribution of tau pathology, the neuronal cell loss, and the disruption of network also differ among early-onset Alzheimer's disease variants (Fig. 1).

Accordingly, atypical Alzheimer's disease variants were added to the revised diagnostic criteria for Alzheimer's disease successively in 2010, 2011 [4], and 2014. In the latest updated version [5], the diagnosis of atypical Alzheimer's disease requires the presence of (1) a clinical phenotype consistent with one of the atypical presentations (visual/posterior variant, logopenic variant of primary progressive aphasia, and frontal variant) and (2) biological, genetic, and/or in vivo

3 Neurology Departement, Pitié-Salpêtrière Hospital, Institut de la mémoire et de la maladie d'Alzheimer, IM2A, Reference Centre for Rare Dementias and Early Onset Alzheimer's Disease, Paris, France

4 Institut du cerveau et de la moelle épinière, Frontlab ICM, INSERM U 1127, CNRS UMR 7225, Sorbonne Universités, Pitié-Salpêtrière Hospital, Paris, France

5 Université de Paris, PARCC, INSERM, In vivo Imaging Research, 75015 Paris, France

6 Radiology Department, APHP Centre, Hôpital Européen Georges Pompidou, 75015 Paris, France 

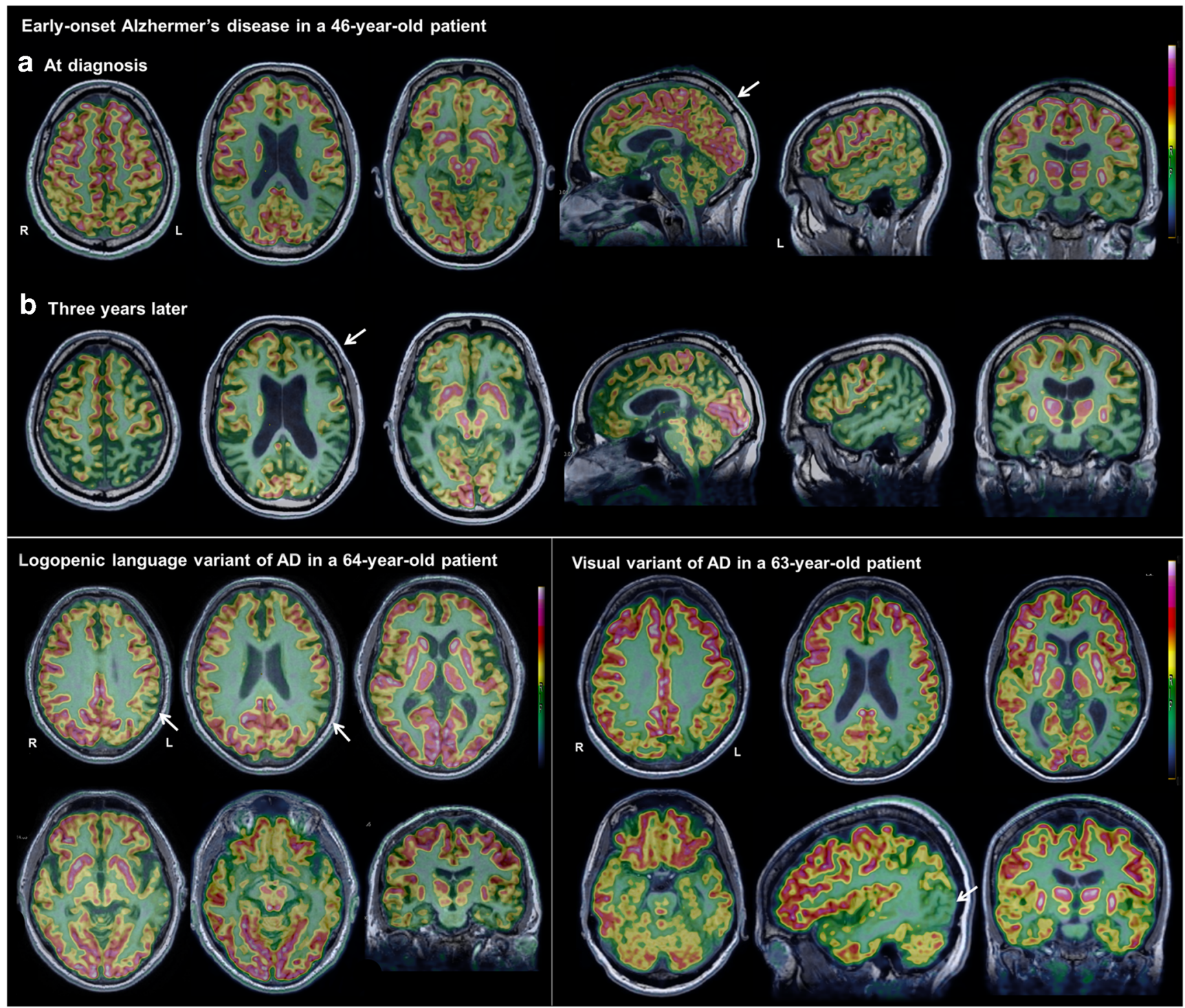

Fig. 1 Representative brain FDG-PET/MRI scans of patients with atypical AD. FDG-PET overlaid on T1-weighted MRI in axial, sagittal, and coronal views; sagittal sections display the left hemisphere. Upper panel: Early-onset AD in a 46-year-old man with 2 years of symptoms duration and CSF tau and amyloid- $\beta$ levels suggestive of $\mathrm{AD}$. (A) At the time of diagnosis, MRI demonstrated a moderate atrophy in the parietal cortex with no hippocampal atrophy, whereas FDG-PET showed a severe cortical hypometabolism, particularly in the bilateral temporoparietal cortex including the precuneus (arrow). (B) 3 years later, cortical atrophy and hypometabolism involved massively the posterior cortex and extended through the left prefrontal cortex (arrow). Bottom right panel: Logopenic variant of primary progressive aphasia in a 64-yearold man with 2 years of disease duration and a CSF biomarker profile suggestive of AD. Neuroimaging shows focal atrophy and decreased metabolism in the left temporoparietal junction (arrow) as well as an accentuated atrophy of the left perisylvian cortex. Hippocampi are relatively preserved. Bottom left panel: Visual variant (also called posterior cortical atrophy) in a 63-year-old woman with symptoms duration of 4 years and a positive CSF AD biomarker profile. The relative preservation of hippocampi is accompanied by accentuated atrophy and severe left > right hypometabolism of the posterior associative cortex, particularly in the occipital cortex (arrow). Altogether, these examples show specific regional imaging phenotypes of early-onset atypical AD patients correlated with their clinical presentation, namely the occipital for the visual variant and the leftlateralized temporal cortices for the logopenic language variant. As illustrated by Sala et al., the parietal cortex is nearly always damaged, irrespective of clinical presentation. This suggests a specific vulnerability of parietal areas especially in atypical AD whereas hippocampi are relatively preserved. L, left; R, right; AD, Alzheimer's disease. Unpublished data by A.K. and R.M.

syndrome [7]. Those new clinical variants add to the taxonomy of Alzheimer's disease, highlight a large variability among patients, and importantly pose major challenges for the diagnosis of early-onset Alzheimer variants [8], emphasizing the importance of in vivo biomarkers for diagnosis. 


\section{Neuroimaging of early-onset Alzheimer's disease}

Over the last two decades, neuroimaging has been of capital importance to support underlying pathophysiological assumptions about the disease and has largely contributed to the evolution of diagnostic approaches. Three well-established neuroimaging markers are now included in the revised criteria for amnestic (typical) forms of Alzheimer's disease [5]: hippocampal atrophy assessed by MRI, temporoparietal hypometabolism shown by FDG-PET, and increased brain amyloid deposition using fibrillar amyloid PET. Importantly, access to imaging biomarkers greatly increases the probability of posing Alzheimer's disease diagnosis, even in preclinical/ predementia stages [5].

A pressing challenge for neuroimaging is to decipher the complexity of Alzheimer's disease by identifying the neural networks involved with anatomical, functional, and clinical variants [9]. In typical Alzheimer's disease, the patterns of brain damage strongly map a resting state fMRI network called the default mode network, whose major nodes include the posterior cingulate cortex and precuneus, the medial prefrontal cortex, and the inferior parietal cortex. The default network is typically impaired in late-onset Alzheimer's disease patients. Conversely, in earlyonset patients, the loss of functional connectivity is greater in other networks than in the default network, e.g., the language network in logopenic language variants, and the higher visual network in visual variants [10]. These observations suggest that Alzheimer's disease pathology possibly originates in a common network, most likely the default mode network anatomically centred on the parietal lobes, and that clinical heterogeneity may reflect the different pathways of pathological propagation from the default mode network into distinct "off-target" functional networks [10, 11].

Can molecular PET imaging help to evidence the phenotypic diversity of Alzheimer's disease and reveal if and how the regional distribution of the pathological $\beta$-amyloid and tau proteins explain the clinical expression of Alzheimer's disease? So far, amyloid PET studies of typical or atypical early-onset of Alzheimer's disease patients have shown diffuse cortical $\beta$-amyloid deposits, regardless of the clinical presentation, and have not demonstrated a correlation between the cognitive profile, metabolic changes, and the distribution of the pathological protein. Moreover, no distinct regional pattern between focal and diffuse Alzheimer's forms has been found with this family of radiotracers $[12,13]$. In contrast, PET tracers targeting tau have demonstrated a close correlation between the distribution of tau deposits and the clinical phenotype. Furthermore, the cerebral distribution of pathological tau protein mirrors the metabolic patterns observed with fluorodeoxyglucose (FDG), the workhorse of PET imaging: hypometabolism is pathologically a close consequence of intracellular pathological tau deposits $[13,14]$. In brief, while the distribution of $\beta$-amyloid is diffuse, the location and density of tau accumulation is regionally correlated with the cognitive symptoms, cerebral blood flow, atrophy, and metabolic changes in early-onset Alzheimer variants [13].

\section{Translation to the clinical practice}

Another challenge for neuroimaging is to benefit routine clinical practice. To this end, the results of neuroimaging research must be translated from group studies to individual patients. In this issue of The European Journal of Nuclear Medicine and Molecular Imaging, Sala and coworkers [15] explore the patterns of brain hypometabolism using FDG-PET in early and atypical Alzheimer's disease patients, both at the group- and single-subject level. They show that each clinical phenotype is associated with group-specific brain regions and networks, namely in the occipital, left-side, or frontal brain regions. Moreover, they obtain a remarkably high consistency of the metabolic patterns with the clinical presentation at the singlesubject level and confirm that the parietal lobes are almost always damaged (in more than $90 \%$ of patients), irrespective of the clinical presentation [16].

By transposing their results from groups to single subjects, Sala and collaborators [15] emphasize the power of PET imaging for clinical practice. They show that group-definedspecific patterns of brain hypometabolism are applicable to study individual Alzheimer's disease patients. Moreover, they evidence that metabolic patterns acquired at the prodromal stage can discriminate among Alzheimer's disease variants, as well as between typical Alzheimer disease and nonAlzheimer dementia conditions (i.e., Lewy body dementia and behavioural variant of frontotemporal dementia). These findings support FDG-PET as a relevant imaging biomarker for diagnostic and prognostic purposes in clinical practice.

Longitudinal follow-up of the dynamic patterns of Alzheimer's disease biomarkers, such as grey matter loss and in vivo PET imaging of tau, metabolism, and amyloid deposits, can contribute significantly to understand the evolution of earlyonset atypical forms of AD [17]. Atypical forms differ from amnestic Alzheimer disease not only because they first hit different sites of the brain but also because they show different spatial and temporal patterns in the progression of brain damage [18]. Along this line, Vanhoutte et al. [19] suggested that the decline of glucose metabolism in amnestic forms progresses along an anterior-to-posterior axis, whereas in non-amnestic forms, it progresses along a posterior-to-anterior axis. Recently, a 1-year follow-up study of Alzheimer's disease variants combining MRI, tau, and amyloid PET [17] found a similar cortical pattern of $A \beta$ deposition that did not change over time. In contrast, higher tau burdens at baseline in the frontal, parietal, and occipital regions correlated with larger atrophy of these regions a year later. Interestingly, tau accumulation and atrophy presented different 
regional patterns: while MRI showed that atrophy predominated in the temporoparietal and occipital cortices, pathological tau deposits spread into the frontal lobes, suggesting a temporal disconnection between protein deposition and neurodegeneration.

The results reported in this issue by Sala and collaborators [15] raise the hope to improve our diagnostic performance of atypical Alzheimer's disease variants in individual patients. They must now be confirmed and, given the rarity of atypical Alzheimer's disease patients, call for a collective effort to build a global database and federate studies from different teams. This will boost our understanding of the diversity of the clinical presentations and evolutions of Alzheimer's disease. Combining multiple imaging modalities [20], such as tau and FDG-PET with fMRI (which can be done simultaneously using PET-MRI), would allow to explore the consequences of pathological deposits on functional networks and better understand the pathological course of Alzheimer's disease. Importantly, the work of Sala et al. emphasizes that, although it is less specific for Alzheimer's disease than "pathophysiological" tracers (i.e., those targeting tau and amyloid), the easily accessible FDG is a powerful and efficient diagnostic tool for the diagnosis of amnestic and atypical Alzheimer's disease, even in prodromal phases. The authors' methodology could also be applied to atypical profiles in older Alzheimer patients, in whom comorbidities greatly affect diagnosis and treatment, in order to disentangle the specific patterns of brain damage due to Alzheimer's disease pathology from those linked to copathologies. In typical late-onset Alzheimer patient, imaging could help to predict the progression from predementia (MCI) to dementia. Ultimately, the methodology used by the authors could be implemented in clinical settings as computer-aided diagnosis.

\section{Compliance with ethical standards}

Conflict of interest The authors declare that they have no conflict of interest.

Statement on published images Images presented are fully anonymized and were collected with the patient's consent in the Nuclear Medicine Department at Pitié-Salpêtrière Hospital.

\section{References}

1. Alladi S, Xuereb J, Bak T, Nestor P, Knibb J, Patterson K, et al. Focal cortical presentations of Alzheimer's disease. Brain. 2007;130:2636-45.

2. Mendez MF. Early-onset Alzheimer disease and its variants: CONTINUUM: Lifelong Learning in Neurology. 2019;25:34-51.

3. Murray ME, Graff-Radford NR, Ross OA, Petersen RC, Duara R, Dickson DW. Neuropathologically defined subtypes of Alzheimer's disease with distinct clinical characteristics: a retrospective study. Lancet Neurol. 2011;10:785-96.

4. McKhann GM, Knopman DS, Chertkow H, Hyman BT, Jack CR, Kawas CH, et al. The diagnosis of dementia due to Alzheimer's disease: recommendations from the National Institute on Aging-
Alzheimer's Association workgroups on diagnostic guidelines for Alzheimer's disease. Alzheimers Dement. 2011;7:263-9.

5. Dubois B, Feldman HH, Jacova C, Hampel H, Molinuevo JL, Blennow $\mathrm{K}$, et al. Advancing research diagnostic criteria for Alzheimer's disease: the IWG-2 criteria. Lancet Neurol. 2014;13: 614-29.

6. Bera G, Migliaccio R, Michelin T, Lamari F, Ferrieux S, Nogues M, et al. Parietal involvement in the semantic variant of primary progressive aphasia with Alzheimer's disease cerebrospinal fluid profile. J Alzheimers Dis. 2018.

7. Lee SE, Rabinovici GD, Mayo MC, Wilson SM, Seeley WW, DeArmond SJ, et al. Clinicopathological correlations in corticobasal degeneration. Ann Neurol. 2011;70:327-40.

8. Warren JD, Fletcher PD, Golden HL. The paradox of syndromic diversity in Alzheimer disease. Nat Rev Neurol. 2012 [cited 2013 Aug 29]; Available from: http://www.nature.com/doifinder/10. 1038/nrneurol.2012.135

9. Buckner RL, Sepulcre J, Talukdar T, Krienen FM, Liu H, Hedden T, et al. Cortical hubs revealed by intrinsic functional connectivity: mapping, assessment of stability, and relation to Alzheimer's disease. J Neurosci. 2009;29:1860-73.

10. Migliaccio R, Gallea C, Kas A, Perlbarg V, Samri D, Trotta L, et al. Functional connectivity of ventral and dorsal visual streams in posterior cortical atrophy. J Alzheimers Dis. 2016;51:1119-30.

11. Lehmann M, Madison C, Ghosh PM, Miller ZA, Greicius MD, Kramer JH, et al. Loss of functional connectivity is greater outside the default mode network in nonfamilial early-onset Alzheimer's disease variants. Neurobiol Aging. 2015;36:2678-86.

12. de Souza LC, Corlier F, Habert M-O, Uspenskaya O, Maroy R, Lamari F, et al. Similar amyloid- $\beta$ burden in posterior cortical atrophy and Alzheimer's disease. Brain. 2011;134:2036-43.

13. Ossenkoppele R, Rabinovici GD, Smith R, Cho H, Schöll M, Strandberg O, et al. Discriminative accuracy of [18F]flortaucipir positron emission tomography for Alzheimer disease vs other neurodegenerative disorders. JAMA. 2018;320:1151-62.

14. Ossenkoppele R, Schonhaut DR, Schöll M, Lockhart SN, Ayakta N, Baker SL, et al. Tau PET patterns mirror clinical and neuroanatomical variability in Alzheimer's disease. Brain. 2016;139:1551-67.

15. Sala A, Caprioglio C, Santangelo R, Vanoli EG, Iannaccone S, Magnani G, et al. Brain metabolic signatures across the Alzheimer's disease spectrum. Eur J Nucl Med Mol Imaging. 2019; (in press).

16. Migliaccio R, Agosta F, Rascovsky K, Karydas A, Bonasera S, Rabinovici GD, et al. Clinical syndromes associated with posterior atrophy: early age at onset AD spectrum. Neurology. 2009;73: 1571-8.

17. Sintini I, Martin PR, Graff-Radford J, Senjem ML, Schwarz CG, Machulda MM, et al. Longitudinal tau-PET uptake and atrophy in atypical Alzheimer's disease. NeuroImage Clin. 2019;23:101823.

18. Firth NC, Primativo S, Marinescu R-V, Shakespeare TJ, SuarezGonzalez A, Lehmann M, et al. Longitudinal neuroanatomical and cognitive progression of posterior cortical atrophy. Brain. 2019;142:2082-95.

19. Vanhoutte M, Semah F, Leclerc X, Sillaire AR, Jaillard A, Kuchcinski G, et al. Three-year changes of cortical 18F-FDG in amnestic vs. non-amnestic sporadic early-onset Alzheimer's disease. Eur J Nucl Med Mol Imaging. 2019; Available from: http:// link.springer.com/10.1007/s00259-019-04519-w.

20. Migliaccio R, Federica A, Basaia S, Cividini C, Habert MO, Kas A, et al. Functional brain connectome in posterior cortical atrophy. Neuroimage Clin. in press.

Publisher's note Springer Nature remains neutral with regard to jurisdictional claims in published maps and institutional affiliations. 